\title{
编者按
}

21 世纪的材料科学家和工程师通过开发新一代的先进材料, 试图解决人类面临的诸多挑战. 缩短材料从研发 到应用的时间, 是推动一个具有强大竞争力的制造业和经济增长的原动力. 从材料研发到应用需要漫长的周期, 其主因是材料在研究与开发过程中主要依赖于经验直觉和反复实验的试错法; 旷日持久的实验周期; 理论研究、 材料设计与制备和工程应用等过程通常由不同的团队完成，数据不能有效共享.

随着材料科学理论和计算机技术的发展，部分传统实验可以用更加快速高效的测试方法和计算模拟替代，逐 步建成集高通量实验、高通量计算模拟和智能化数据库管理为一体的材料集成计算创新平台. 美国政府率先启动 了旨在保持其全球竞争力的“材料基因组计划”，该计划预期实现将这些模拟方法、集成工具和相关数据库渗透到 从材料发现、优化、制备到实用化的整个流程，减少实验量，提高预测能力，从而将材料的平均研发周期缩短到目 前的一半.

在 2013 年《科学通报》第 35 期“材料基因组计划”专辑中，我们组织了国内外专家，对材料计算模拟、高通 量实验和数据库建设及管理三大方向进行了探讨 ${ }^{[1 \sim 14]}$. 第一性原理计算在揭示机理、描述物理现象的本质等方面 无可替代, 随着计算精度的提高和计算速度的倍增, 已成为材料领域中的重要研究工具. 但是大多数第一性原理 计算量大耗时，对多元多相体系复杂的晶体及组织结构、有限温度下的材料性质等计算困难更大，无法达到工程 应用所需要的精度. 而计算热力学和计算动力学方法依据材料热力学和相变动力学等理论, 结合由实验数据优化 得到的模型参数集合(即材料基础数据库), 能够计算真实工程材料体系的热力学、动力学及物理性能, 以及模拟相 变和组织演变过程. 将其与第一性原理计算紧密结合, 可最大限度地发挥各自的优势. 上述计算方法属于多尺度 集成计算中的微观和介观环节, 配合有限元分析方法、计算力学等宏观尺度计算, 可以构建旨在研究多元多相材 料的微观组织结构和性能, 进行材料理性设计的材料集成计算创新平台. 其中材料数据库发挥着重要作用, 而高 质量的数据库必须建立在大量高精度的实验数据以及理论计算数据之上.

建设材料集成计算创新平台，并将其成功地应用到先进材料的设计、生产和使用中，是“材料基因组计划”实 施的初衰. “材料基因组”计算模拟应用”专题的重点在于展示将计算模拟与新型材料开发密切结合的工作. 组稿文 章涉及钢铁材料、 $\mathrm{Ti}$ 合金等重要结构材料(镍基高温合金以及部分功能材料已在前面专辑中讨论)以及生产制备这 些材料必不可少的凝固过程，希望能够抛砖引玉，推动基于“材料基因组”理念的计算模拟在工业生产中的应

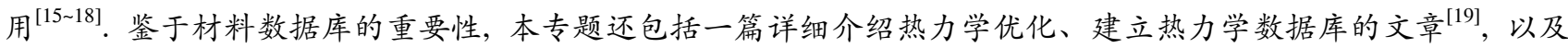
一篇讨论应用第一性原理计算材料力学性能的文章 ${ }^{[20]}$.

\section{朱静 $^{\mathbb{1}}$ ，鲁晓刚 ${ }^{(2)}$ ，王卓 ${ }^{3}$}

(1) 清华大学材料学院, 先进陶瓷和精细工艺国家重点实验室, 先进材料教育部重点实验室, 北京电子显微镜中心, 北京 100084 ;

(2) 上海大学材料科学与工程学院, 上海 200072; (3) 钢铁研究总院, 北京 100081

1 刘梓葵. 关于材料基因组的基本观点及展望. 科学通报, 2013, 58: 3618-3622

2 王绍青, 叶恒强. 晶体材料基因组问题第一原理计算研究. 科学通报, 2013, 58: 3623-3632

3 Agren J. 材料基因组与相图计算. 科学通报, 2013, 58: 3633-3636

4 陈龙庆. 相场模拟与材料基因组计划. 科学通报, 2013, 58: 3637-3641 
5 何燕霖, 鲁晓刚, 朱娜琼, 等. 基于 CALPHAD 方法的摩尔体积建模讨论了基于 CALPHAD 方法, 从纯物质到多元. 科学通报, 2013, 58: 3642-3646

6 赵继成. 材料基因组计划中的高通量实验方法. 科学通报, 2013, 58: 3647-3655

7 鲁晓刚, 王卓, Cui Y W, 等. 计算热力学、计算动力学与材料设计. 科学通报, 2013, 58: 3656-3664

8 司晨, 陈鹏程, 段文晖. 石墨烯材料的电子功能化设计: 第一原理研究进展. 科学通报, 2013, 58: 3665-3679

9 Cui Y W, Xu G L, Chen Y. 计算扩散动力学在稀有金属材料研究和设计中的应用. 科学通报, 2013, 58: 3680-3691

10 赵彦, 张洪宇，韦华，等. 镍基单晶高温合金中 $\gamma^{\prime}$ 相筏化行为的相场法研究进展. 科学通报, 2013, 58: 3692-3703

11 黄智恒, 熊桦, 伍智勇, 等. 半导体集成与封装中基于微观组织的多物理场耦合模拟. 科学通报, 2013, 58: 3704-3716

12 朱静, 于荣. 亚埃分辨与皮米精度原子结构的实验测量与计算. 科学通报, 2013, 58: 3717-3721

13 徐东生, 王睥, 杨锐, 等. 钛铝中 $\langle 011$ ]超位错的非对称形核及运动的分子动力学模拟. 科学通报, 2013, 58: 3722-3732

14 王卓, 杨小渝, 郑宇飞, 等. 材料基因组框架下的材料集成设计及信息平台初探. 科学通报, 2013, 58: 3733-3742

15 黄卫东, 王猛. 凝固过程数值模拟的研究进展和发展趋势. 科学通报, 2014, 59: 845-853

16 常辉, Gautier E, 周廉. 一种亚稳 $\beta$ 钛合金的相变动力学. 科学通报, 2014, 59: 854-858

17 潘涛, 朱静, 杨才福, 等. QLT 处理 $9 \mathrm{Ni}$ 低温钢精细结构的动力学模拟和实验. 科学通报, 2014, 59: 859-865

18 罗海文, 向睿, 陈凌峰, 等. 取向硅钢中脱碳工艺的数值模拟. 科学通报, 2014, 59: 866-871

19 薄宏, 刘立斌, 熊翔, 等. Al-Dy, Dy-Zr 和 Al-Dy-Zr 体系的热力学优化. 科学通报, 2014, 59: 872-879

20 于荣, 张祺, 詹倩, 等. 弹性软模决定材料硬度. 科学通报, 2014, 59: 880-886 